\title{
THE DIAGNOSTIC CAPABILITIES OF THE CBCT IN VERIFICATION THE PATHOLOGIES OF BITE AND POSITIONS OF INDIVIDUAL TEETH ${ }^{1}$
}

\section{Babkina T. M., Storozhchuk Yu. O.}

\section{INTRODUCTION}

Today, the dentist plays an important role in improving the quality and effectiveness of primary care at the pre-hospital stage, especially in rural areas. The work of a dentist in the field of primary health care, integrated with a general practitioner - family doctor, to improve the quality and effectiveness of diagnostic, prevention, treatment and rehabilitation care to the population of different age groups plays a significant role.

In most European countries, where $80-85 \%$ of dentists are general practitioners, they work at the primary level on the principle of a family doctor, treating the most common diseases of the teeth and mouth and carrying out preventive measures. However, it is difficult to imagine the recognition and treatment of various diseases of the maxillofacial region without modern diagnostic methods, including radiological, which are the leading methods in dental practice.

Focusing on the current state of radiology, it should be noted that the method of cone-beam computed tomography is becoming a standard in dental practice, so the use of cone-beam computed tomography in orthodontic practice can be classified into four areas of application:

1) proper diagnostics: evaluation of skeletal features, symmetry or asymmetry of bone and dental structures, evaluation of the position of displaced and impacted teeth, bone growth of the facial skeleton, patency of the upper respiratory tract and complex structures of the temporomandibular joint, as well as areas of the hard palate;

2) treatment planning: different volume of orthodontic interventions, prior to fixing braces, precise location in the area of unerupted or impacted teeth, for the manufacture of various types of orthodontic devices in conjunction with the capabilities of CAD/CAM technologies;

${ }^{1}$ The article is a fragment of the "Algorithm of radiation diagnosis in secondary adentia and planning of dental implantation” research, state registration № 0114U002212.

The author declares no conflict of interest. 
3) monitoring the dynamics of changes in the dentofacial system during and after treatment: the results of augmentation and reconstruction of skeletal elements, during the superimposition of the original and changed position of individual structures of the facial skeleton;

4) risk assessments: case study of orthodontic-associated paresthesia, root resorption symptoms due to orthodontic displacement and changes in the structure of the temporomandibular joint that occur after comprehensive dental rehabilitation $2,3,4,5,6$.

According to the recommendations of the American Academy of Oral and Maxillofacial Radiology, CBCT in orthodontic practice is recommended for use: 1) for localization of the position of displaced and impacted teeth and identification of associated resorption of the tooth; 2) in cases of cleft lip and palate; 3 ) in cases of using mini-implants (to determine the height and width of the alveolar process); 4) in cases of severe skeletal disorders (for orthosurgical treatment planning); 5) to plan surgery for removing impacted and displaced teeth; 6) to assess the status of the temporomandibular joint. At the same time, the SedentexCT Project European evidence-based recommendations on a similar issue somewhat limit the use of this technology but do not deny the use of cone-beam tomography in individual clinical cases.

The data of the study conducted by Tarazone and colleagues (2011) indicate that the efficiency of using cone-beam computed tomography (CBCT) to register such indicators as the length of the dental arch, mesiodistal dimensions of the teeth and the width of the dental arch in the molars and canines, is similar to the analysis carried out on the basis of digitized plaster models ${ }^{7}$. The difference between the results obtained in the course of digital scanning and cone-beam

${ }^{2}$ Agrawal J. M., Agrawal M. S., Nanjannawar L. G., Parushetti A. D. CBCT in orthodontics: the wave of future. J Contemp Dent Pract. 2013. 14(1). P. 153-157.

3 Almuzian M., Almukhtar A., O’Neil M., Benington P., Al Anezi T., Ayoub A. Innovation in prediction planning for anterior open bite correction. Australian orthodontic journal. 2015. 31(1). P. 78.

${ }^{4}$ Kapila S. D., Nervina J. M. CBCT in orthodontics: assessment of treatment outcomes and indications for its use. Dentomaxillofacial Radiology. 2014. 44(1). P. 20140282.

${ }^{5}$ Kapila S., Conley R. S., Harrell W. E. The current status of cone beam computed tomography imaging in orthodontic. Dentomaxillofacial Radiology. 2011. 40(1). P. 24-34.

${ }^{6}$ Silva M. A. G., Wolf U., Heinicke F., Bumann A., Visser H., Hirsch E. Cone-beam computed tomography for routine orthodontic treatment planning: a radiation dose evaluation. American Journal of Orthodontics and Dentofacial Orthopedics. 2008. 133(5). P. 640-e1.

${ }^{7}$ Tarazona B., Llamas J. M., Cibrian R., Gandia J. L., Paredes V. A comparison between dental measurements taken from CBCT models and those taken from a digital method. The European Journal of Orthodontics. 2011. 35(1). P. 1-6. 
studies, according to some authors, is so small that it can be considered clinically acceptable. However, justification of expediency in use of CBCT specifically for diagnostics of pathologies of occlusion and position of separate teeth remains an actual question of practical dentistry.

\section{Study goal}

To determine the advantages and justify the feasibility of using the method of cone-beam computed tomography for verification of malocclusion pathologies and the position of individual teeth.

The method of X-ray examination, which uses a cone-shaped X-ray beam to obtain multiple X-ray images of the patient with the help of computer tomographs has acquired significant relevance. Images are recorded on the sensor in the form of a flat panel or image amplifiers; important aspect is that a three-dimensional image can be obtained only with one revolution of the beam and the sensor, thereby reducing the radiation load on the patient.

CT images of 50 patients aged between 25 and 40 years old (men) and 25 and 45 years old (women) with abnormalities of occlusion and position of individual teeth were analyzed (table 1). Radiological methods of research were carried out on a Planmeca ProPax 3D MID cone-beam computed tomograph with SmartPan system. In order to select patients with relevant pathologies, they were divided into categories. At the same time, the results of CBCT diagnostics, data of description and diagnosis were taken into account (table 2).

Table 1

Group of examined patients

\begin{tabular}{|c|l|c|c|}
\hline № & \multicolumn{1}{|c|}{ Name } & Amount & \% \\
\hline 1 & $\begin{array}{l}\text { Total number of patients } \\
\text { examined }\end{array}$ & $\mathrm{n}=50$ & $100 \%$ \\
\hline 2 & Norm & $\mathrm{n}=7$ & $14 \%$ \\
\hline 3 & Orthodontic pathology & $\mathrm{n}=43$ & $86 \%$ \\
\hline
\end{tabular}

Table 2

Types of research and revealed pathology (nosological entity)

\begin{tabular}{|l|l|c|l|l|}
\hline № & Scan area & $\begin{array}{c}\text { Scan } \\
\text { area size } \\
\text { (DxH } \\
\text { mm) }\end{array}$ & Amount, \% & Nosological entity,\% \\
\hline 1 & $\begin{array}{l}\text { Facial } \\
\text { skull } \\
\text { bones }\end{array}$ & $200 x 170$ & $5(10 \%)$ & $\begin{array}{l}\text { Distocclusion 2 }(0,1 \%) ; \\
\text { Distocclusion and impacted } \\
\text { canines 2 }(0,1 \%)\end{array}$ \\
\hline
\end{tabular}


Table 2 (ending)

\begin{tabular}{|l|l|l|l|l|}
\hline № & Scan area & $\begin{array}{c}\text { Scan } \\
\text { area size } \\
(\mathbf{D x H} \\
\mathbf{m m})\end{array}$ & Amount, \% & \multicolumn{1}{|c|}{ Nosological entity, \% } \\
\hline 2 & Segment & $8 \times 5$ & $10(20 \%)$ & $\begin{array}{l}\text { Impacted and displaced } \\
\text { teeth 10 (5\%); } \\
\text { Maxillary sinus cysts } \\
\text { (comorbidity) 4 (0,4\%) }\end{array}$ \\
\hline 3 & $\begin{array}{l}\text { Upper and } \\
\text { lower jaw }\end{array}$ & $100 \times 100$ & $35(70 \%)$ & $\begin{array}{l}\text { Distocclusion 29 (10,15\%); } \\
\text { Distocclusion and pathology } \\
\text { of the nasal cavity 10 } \\
(4,14 \%) ; \\
\text { Distocclusion and bilateral } \\
\text { maxillary sinusitis 5 (2,9\%) }\end{array}$ \\
\hline & & & & $\begin{array}{l}\text { Mesocclusion 6 (4,9\%) } \\
\text { Mesocclusion and impacted, } \\
\text { displaced wisdom teeth } \\
4(0,8 \%)\end{array}$ \\
\hline & & & &
\end{tabular}

\section{Results and discussion}

The specificity of the use of CBCT among orthodontic patients is that the use of this method is limited by the relatively large radiation dose compared to the X-ray of the skull, but at the same time, it is smaller than when using spiral tomography. Due to the low contrast resolution, the process of comparing soft tissue changes with CBCT is difficult, although a number of additional diagnostic techniques can be used to diagnose these aspects. The lack of standardized approaches to 3D imaging in different age groups also complicates the process of adequate diagnostic and at the same time reasonable approach to the verification of various orthodontic pathologies. In addition, in most cases, when monitoring the dynamic changes of the dentofacial system, it is advisable to choose constant bone points, in respect of which the comparison of the initial conditions of the diagnostic situation and treatment planning is then carried out. This radiological examination method, when used correctly, makes it possible to: conduct primary diagnosis; analyze changes during treatment; carry out control at the final stage of orthodontic treatment. It is also possible to reform CBCT data into a cephalometric image. Accordingly, there is an opportunity to evaluate a number of auxiliary orthodontic indicators that are used to verify the complexity of sagittal pathology and to determine its dental or skeletal etiology (Fig. 1). 

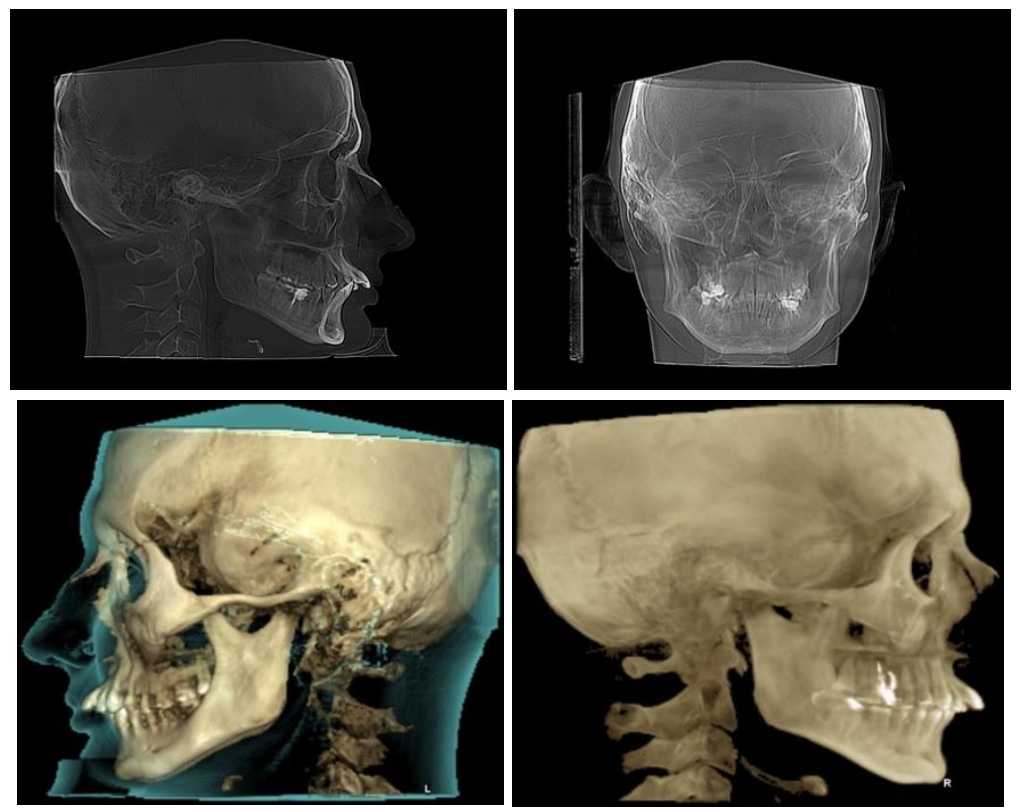

Fig. 1. Principle for software-based telerentgenogram generation from data obtained during CBCT study

In order to substantiate the feasibility of using the CBCT method to provide an objective determination of the parameters of malocclusion and the condition of displaced or impacted teeth, the results of the analysis of 50 tomographs from patients who were previously selected on the basis of the presence of dental pathology will be presented below.

Among all 50 patients selected for analysis of diagnostic results, $5(10 \%)$ of them, in addition to CBCT data from a complete study of the head area, also had results of radiological diagnostics of the sinuses in the habitual occlusion. In these $5(10 \%)$ patients distocclusion was detected in $2(0,1 \%)$ patients, in other $2(0,1 \%)$ the pathology of distocclusion was accompanied by impacting of individual teeth (canines) and the corresponding radiological signs of pathology in the maxillary sinuses. In one of these patients, it was not possible to detect pathology of occlusion on the CBCT, but cystogranuloma was observed in the tooth 2,5 area.

In $10(5 \%)$ of the 50 pre-selected CBCT data, the results of the study were presented in the form of segments (areas of interest), while all had impacted and displaced canines and $4(0,4 \%)$ patients had associated pathology (cysts in the right maxillary sinus). 
In $35(17,5 \%)$ patients, the results of CT diagnostics were presented in the form of tomographic examination of both jaws. In $29(10,15 \%)$ of these patients, distocclusion was observed (among them, $10(4,14 \%)$ cases were associated with changes in the nasal septum, and $5(2,9 \%)$ - with bilateral maxillary sinusitis, in $6(4,9 \%)$ - mesocclusion was observed (among them, $4(0,8 \%)$ cases associated with displaced and impacted wisdom teeth). This high prevalence of impacted and displaced canines has been noted in a number of preliminary studies. According to Sajnani and colleagues (2012) ${ }^{8}$, Arabion and colleagues (2017) ${ }^{9}$, the prevalence of impacted and displaced canines is about $1-3 \%$, but in comparison with these indicators of other teeth, it is less only in the prevalence of impacted upper and lower third molars.

A definitive link between malocclusion pathology and the influence of existing displaced and impacted dentition units has not yet been established, although the interdependence of these types of disorders can still be traced in a number of previously published articles.

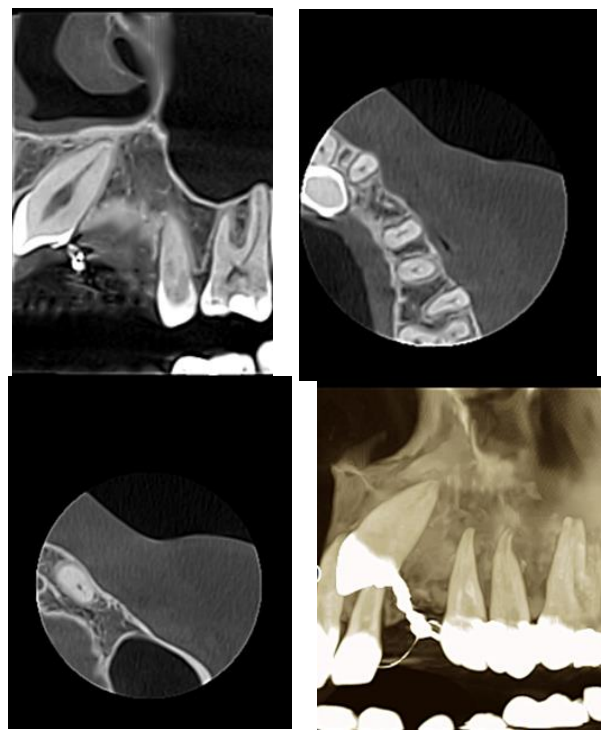

Fig. 2. Clinical case № 1

${ }^{8}$ Sajnani A. K., King N. M. Prevalence and characteristics of impacted maxillary canines in southern Chinese children and adolescents. Journal of investigative and clinical dentistry. 2014. 5(1). P. 38-44.

${ }^{9}$ Arabion H., Gholami M., Dehghan H., Khalife H. Prevalence of Impacted Teeth among Young Adults: A Retrospective Radiographic Study. Journal of Dental Materials and Techniques. 2017. 6(3). P. 131-137. 
On fig. 2 the results of CBCT diagnosis of 26-year-old patient M. at the stage of orthodontic treatment are shown. Impacted tooth 2,3 and its displacement to the area of teeth 2,2 and 2.1 are clearly visualized on the CBCT sections. The absence of orthodontic treatment in this case can potentially provoke a change in the position of the frontal teeth, which in turn will cause not only a violation of the existing occlusive ratio (due to non-physiological load on the periodontal tissues and resorption of the surrounding bone tissue of the alveolar ridge), but also alteration of the aesthetic profile of the smile with the possibility of marginal gum reduction due to periodontal complications.

The possibility of implementing such predictions according to orthopantomography and spot radiography is limited since there are a summation effect and the inability to assess the spatial position of the impacted canine. Digital rendering (3D modeling) of the image allows you to refine the anatomical picture, which also greatly simplifies the process of interpreting the clinical situation for the patient by the doctor.

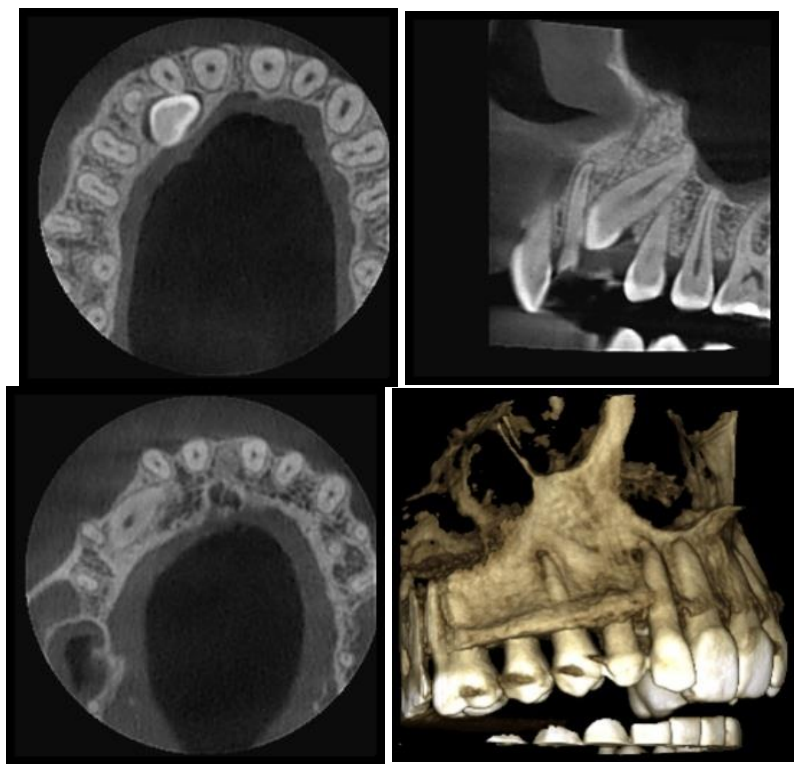

Fig. 3. Clinical case № 2

Fig. 3 shows the results of CBCT-diagnosis of a 26-year-old patient K., who has a clinical situation similar to that described in clinical case № 1 (Fig. 2) but displaced and impacted tooth 1.3 in this patient is observed in 
parallel with retention of milk canine (tooth 5.3). The delay in resorption of the canine milk root caused a violation of the normal position of the tooth 1.3. Further orthodontic treatment plan should take into account the availability of space in the dentition for adequate positioning of the permanent tooth and the potential risks that may arise during its orthodontic extrusion.

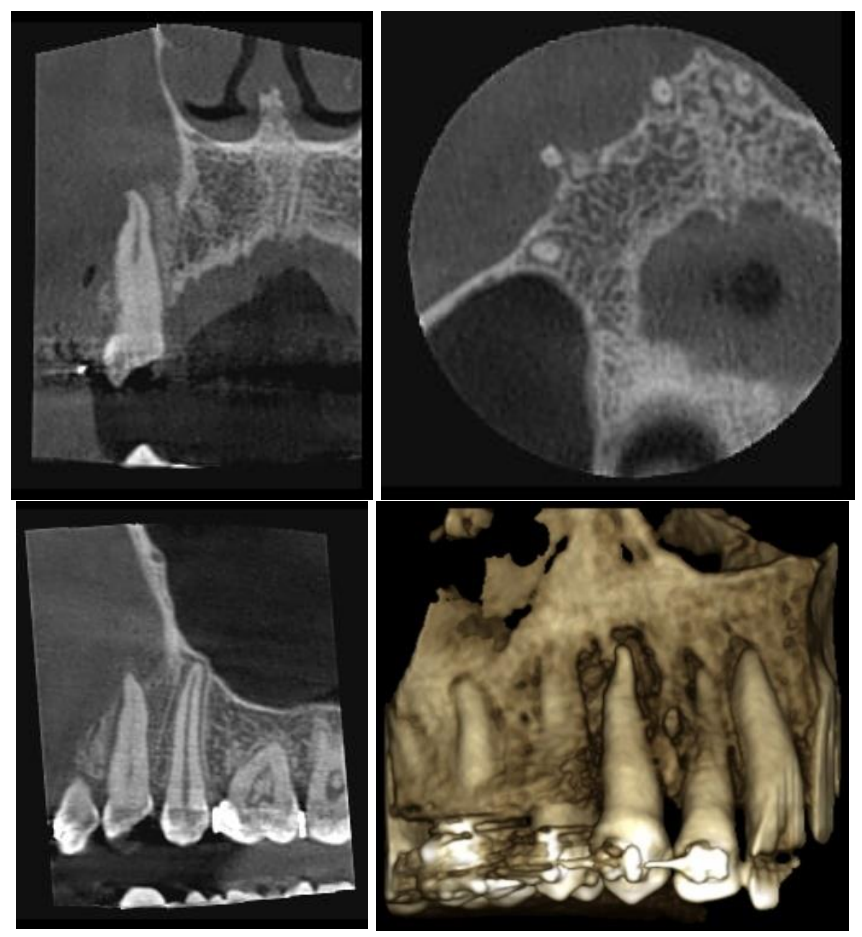

Fig. 4. Clinical case № 3

Fig. 4 shows the results of CBCT diagnosis of 28-year-old patient P. after treatment due to tooth 1,3 displacement. The level of bone resorption from the vestibular side of the canine is more than half the root. Further treatment of the patient should include the need to restore the bone tissue of the tooth through the procedure of solid-tissue augmentation, as well as the possible need for correction of soft tissue in the area of the problem tooth, since such a significant reduction of bone tissue is often associated with the loss of the marginal gum, which in turn compromises the aesthetic profile of the patient's smile. 

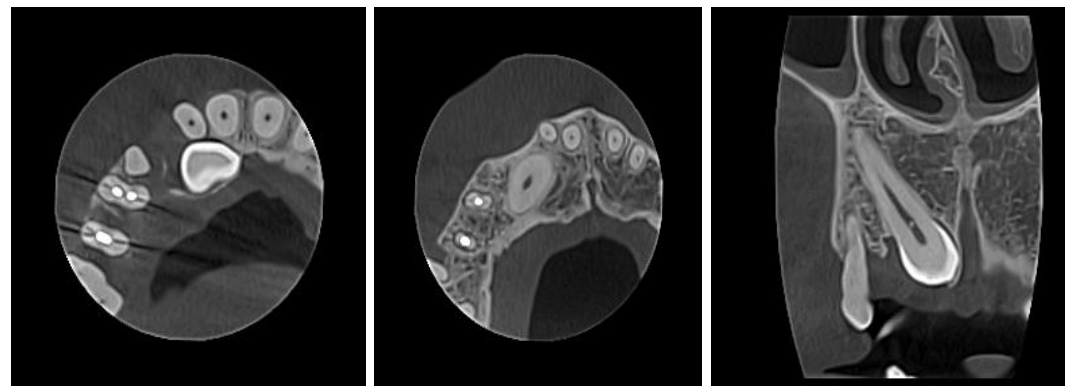

Fig. 5. Clinical case № 4

Pat. M., 28 years old, tooth retention 1,3 , tooth 5,3 - in the dentition. CBCT sections axial, coronal. The image identifies a retentive, distoped tooth 1,3 that closely adjoins the wall of the incisor canal. Based on this examination technique, it is possible to plan orthodontic treatment more precisely.
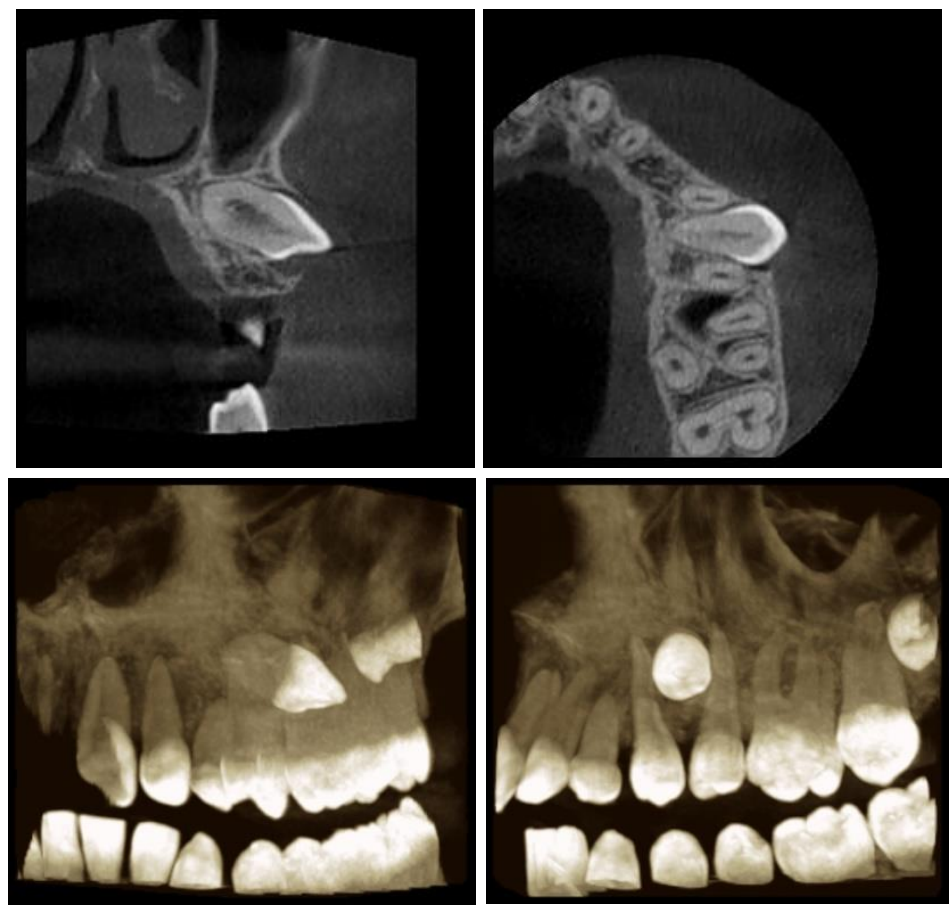

Fig. 6. Clinical case № 5 
Fig. 6 shows a CBCT examination of a patient aged 27 years. According to CBCT data (axial, coronal sections and virtual 3D model), a retarded, distoped tooth is visualized 2,3. To all of the above, the tooth is in transposition, that is, the position of tooth 2,3 is incorrect, the anomalon is wrong, as if the teeth had changed places in the alveolar process and dentition. The periodontal gap is not traced throughout. In the alveolar process of the upper jaw of the tooth 2,3 is located horizontally. The tooth is located in the interdental septum 2,4,2,5. The vestibular surface of the tooth is located above, palatine - from below. The crown of the tooth is directed vestibularly; the crown part is directed palatally. The periodontal fissure on the palatine surface is not observed in places (ankylosis). The cutting edge of the tooth crown in the teething stage, violates the integrity of the cortical plate. The crown is located under the mucosa. The apical third of the root is intimately adjacent to the lower wall of the maxillary sinus.
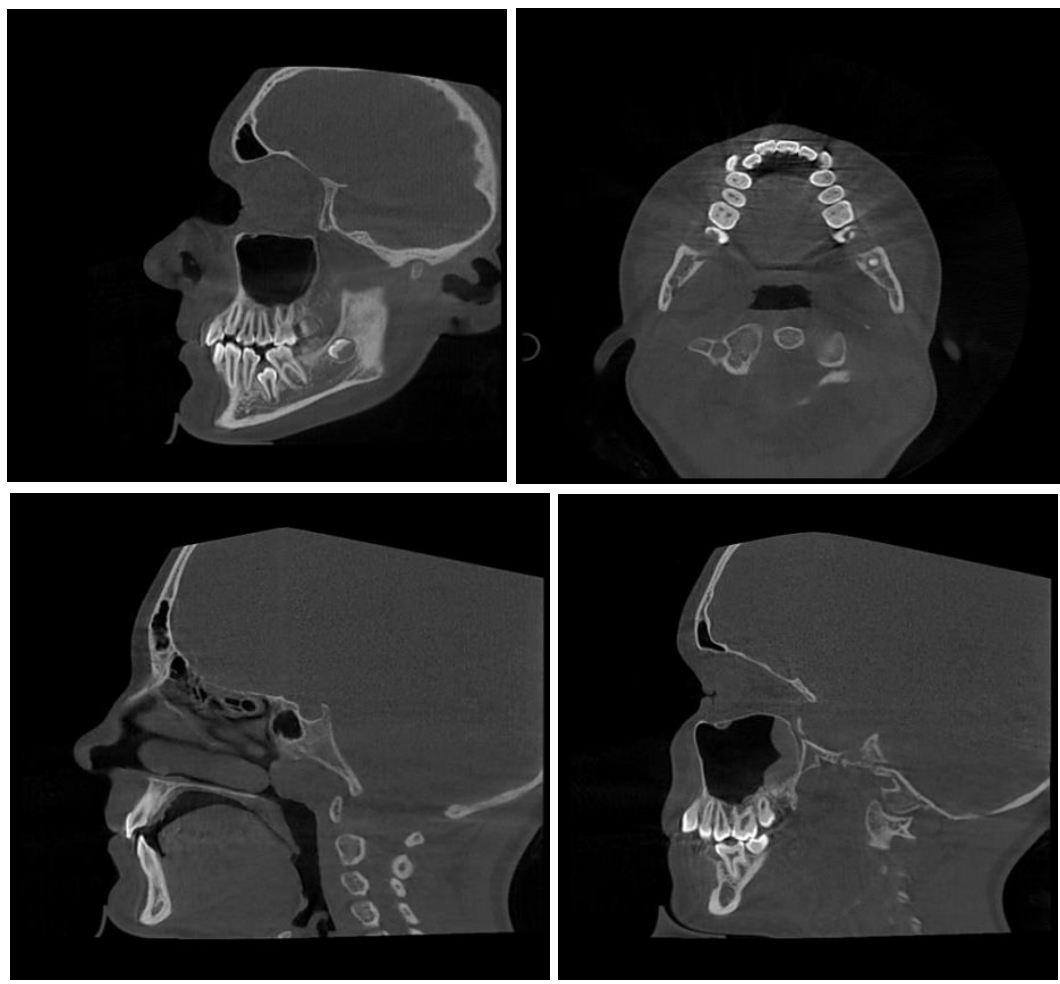

Fig. 7. Clinical case № 6 
On sections, a CBCT study of a 13-year-old patient $\mathrm{P}$ is presented. On the axial, sagittal sections, a retarded, distoped tooth 3,5 is visualized, which is located in the alveolar protrusion of the lower jaw, vertical position. The apex of the tooth root is in the formation stage, intimately adjacent to the upper wall of the mandibular canal. In the area of the upper jaw, vestibularly displaced teeth $1,3,2,3$ are determined, tooth 1,2 is slightly displaced palatally. These changes are due to crowding of the frontal group of teeth of the upper jaw.
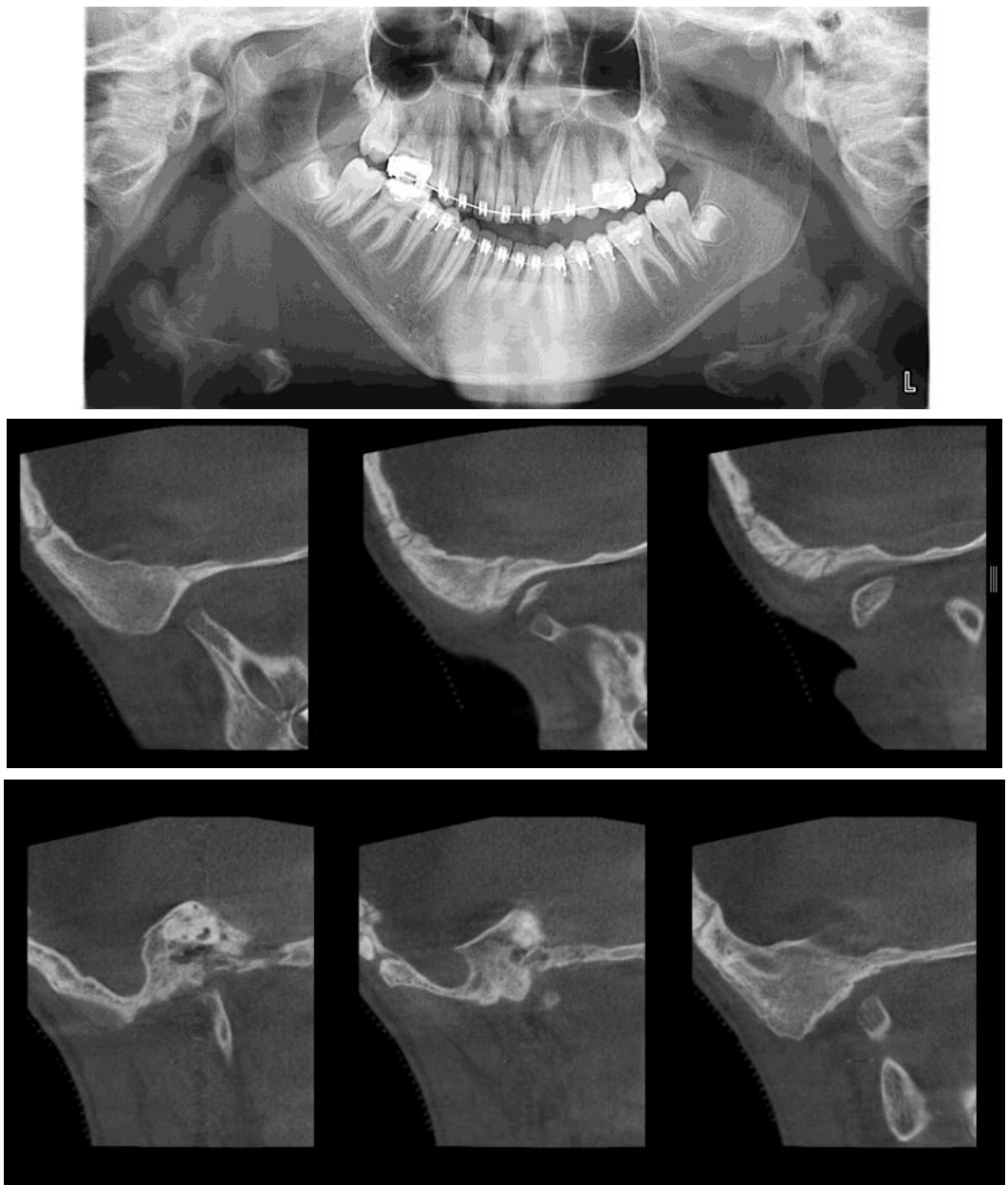

Fig. 8. Clinical case № 7 
Crowding of teeth is a pathology characterized by a close position and tooth rotations. Due to the fact that the teeth are too close to each other, some of them erupt outside the arc and overlap each other. Crowding can form on both one and both jaws, as well as simultaneously in the anterior and lateral sections. In addition, the curvature, if left untreated, worsens over time, which leads to very sad consequences and requires more serious treatment. An interesting fact is that crowding of the lower teeth occurs several times more often than in the upper ${ }^{10}$.

So, as the patient was examined throughout the maxillofacial region, we were able to identify concomitant CT symptoms. Hypertrophy of the pharyngeal tonsil, which narrows the lumen of the nasopharynx, by about $1 / 2$.

In addition to the enlarged pharyngeal tonsil, a local thickening of the mucous membrane of the maxillary sinus along the posterolateral wall is visualized in sections.

Thanks to the correctly selected screening technique, this patient was able to identify the underlying disease, as well as the associated pathology.

Patient From 14 years old at the stage of orthodontic treatment: in the area of the dentition of the upper and lower jaw, the bracket system is determined. X-ray orthopantomogram data were not enough to determine the anatomical features of the temporomandibular joint. So, as in the patient's history, an anomaly of the development of TMJ on the right, they performed $\mathrm{CBCT}$ of this anatomical region. At the multiplanarone reconstruction of the TMJ, it was possible to study in detail the shortened branch of the lower jaw, the displacement of the condylar condyle head down, its underdevelopment.

According to Suomalainen A. (2015) and colleagues ${ }^{11}$, despite the fact that the methods of orthopantomography and spot-film radiography of the dentofacial system are still the most common in dental practice, the tendency to improve existing approaches to the treatment of various types of dental pathologies justifies the need for more frequent use of CBCT as the most objective method of verifying the state of the maxillofacial area. This method provides the ability to diagnose simultaneously in several critical anatomical and functionally significant areas, based on a single set of $* \mathrm{dcm}$ data. In addition, Almuzian M. and colleagues (2015) even described an innovative approach for predicting malocclusion treatment results using the diagnostic capabilities of CBCT: the authors proposed to use the results of $\mathrm{X}$-ray diagnosis not only for a proper cephalometric analysis after selecting

\footnotetext{
${ }^{10}$ Crowding of teeth - can it be fixed? URL: https://www.startsmile.ru/ortodontiya/ skuchennost-zubov/.

${ }^{11}$ Suomalainen A., Esmaeili E. P., Robinson S. Dentomaxillofacial imaging with panoramic views and cone beam CT. Insights into imaging. 2015. 6(1). P. 1-16.
} 
the desired projection from CBCT, but also for getting valid threedimensional models of the facial skeleton structure during the complex of iatrogenic interventions, which can actually be used for planning all necessary orthognathic surgery given the potential displacements of bone markers. In the final stage of treatment, the authors also propose to use the principle of superimposition of initial and final results, which are diagnostic areas of the face on CBCT, to monitor and analyze the level of effectiveness achieved from the point of view of occlusion correction, interfissural ratios correction and changing the position of the upper jaw in the structure of the facial skeleton. In addition, the acquisition of CBCT data with a wide field of shot allows monitoring the temporomandibular joint condition, which makes registration of potential complications that may occur during orthodontic treatment possible.

Limitations on conducting large-scale studies may be related to the output parameters of a given area of interest, which are associated with both the capabilities of the CBCT equipment and the regulation of the derivative by the operator, in which cases additional research should be justified on the basis of its prospective advantages to carry out diagnostics and treatment taking into account the potential risks of additional radiological irradiation (according to the ALARA principle - As Low As Reasonably Achievable).

\section{CONCLUSIONS}

In the course of the analysis of radiological studies data obtained from dental patients with malocclusion and displacement of individual teeth, we found that:

1. The use of the CBCT method allows to expand the possibilities of primary diagnosis of the above pathologies and to objectify the degree of complexity of the disturbed relationship of the dental arches, as well as impacting and displacement of the corresponding units of dentition.

2. Function of software-based generation of teleroentgenogram from this .dcm-data set allows analyzing the necessary orthodontic indicators in the appropriate projection.

3. The possibility of simultaneous diagnosis of the maxillary sinus area and the temporal and mandibular area substantiates the feasibility of using CBCT to provide the most comprehensive approach to the rehabilitation of dental patients, thus ensuring the overall quality of dental care.

4. The reasonable use of CBCT allows not only to objectify the condition of impacted and displaced units of the dentition, but also to predict the risk of potential complications and to help in the choice of the most reasoned method of treatment taking into account the criterion of urgency to carry out treatment and preventive measures. 


\section{SUMMARY}

This paper discusses issues of X-ray diagnostics of dental arches ratio anomalies and anomalies of teeth position in patients aged 25-45 years old. Study goal. To assess the specifics of the use of cone beam computed tomography (CBCT) among orthodontic patients and to determine the correct use of this technique. Materials and methods. CT images of 50 patients aged between 25 and 40 years old (men) and 25 and 45 years old (women) with abnormalities of occlusion and position of individual teeth were analyzed. Radiological methods of research were carried out on a Planmeca ProPax 3D MID cone-beam computed tomograph with SmartPan system. Results. Evaluating the data obtained, it was found that CBCT provides an opportunity to conduct primary diagnosis, analyze changes during treatment and conduct control at the final stage of orthodontic treatment, as well as to generate a cephalometric image reformatted from the CPCT data. Accordingly, it is possible to evaluate a number of auxiliary orthodontic indicators used to check the complexity of sagittal pathology and determine its dental or skeletal etiology. Conclusions. During the analysis of dental patients with malocclusion pathologies and pathologies of individual tooth positions, it was found that the use of CBCT allows to expand the possibilities of primary diagnosis of the abovementioned pathologies and to maximize the degree of objective assessment of the dental arches ratios, as well as the impacting and displacement of the corresponding teeth. Function of the program, which allows to provide teleroentgenogram from obtained*.dcm-data set to analyze the necessary orthodontic indicators in the appropriate projection, which in turn makes possible simultaneous diagnosis of the maxillary sinuses and temporomandibular joint, justifies the feasibility of using the tomographic method to provide the most comprehensive approach to the rehabilitation of dental patients, thereby ensuring the best overall quality of dental care.

\section{REFERENCES}

1. Agrawal J. M., Agrawal M. S., Nanjannawar L. G., Parushetti A. D. CBCT in orthodontics: the wave of future. J Contemp Dent Pract. 2013. 14(1). P. 153-157.

2. Almuzian M., Almukhtar A., O’Neil M., Benington P., Al Anezi T., Ayoub A. Innovation in prediction planning for anterior open bite correction. Australian orthodontic journal. 2015. 31(1). P. 78.

3. Kapila S. D., Nervina J. M. CBCT in orthodontics: assessment of treatment outcomes and indications for its use. Dentomaxillofacial Radiology. 2014. 44(1). P. 20140282. 
4. Kapila S., Conley R. S., Harrell W. E. The current status of cone beam computed tomography imaging in orthodonti. Dentomaxillofacial Radiology. 2011. 40(1). P. 24-34.

5. Silva M. A. G., Wolf U., Heinicke F., Bumann A., Visser H., Hirsch E. Cone-beam computed tomography for routine orthodontic treatment planning: a radiation dose evaluation. American Journal of Orthodontics and Dentofacial Orthopedics. 2008. 133(5). P. 640-e1.

6. Tarazona B., Llamas J. M., Cibrian R., Gandia J. L., Paredes V. A. comparison between dental measurements taken from CBCT models and those taken from a digital method. The European Journal of Orthodontics. 2011. 35(1). P. 1-6.

7. Sajnani A. K., King N. M. Prevalence and characteristics of impacted maxillary canines in southern Chinese children and adolescents. Journal of investigative and clinical dentistry. 2014. 5(1). P. 38-44.

8. Arabion H., Gholami M., Dehghan H., Khalife H. Prevalence of Impacted Teeth among Young Adults: A Retrospective Radiographic Study. Journal of Dental Materials and Techniques. 2017. 6(3). P. 131-137.

9. Crowding of teeth - can it be fixed? URL: https://www.startsmile.ru/ ortodontiya/skuchennost-zubov/.

10. Suomalainen A., Esmaeili E. P., Robinson S. Dentomaxillofacial imaging with panoramic views and cone beam CT. Insights into imaging. 2015. 6(1). P. 1-16.

\section{Information about authors:}

Babkina T. M.,

Doctor of Medical Sciences, Professor, Head of the Department of Radiology Shupyk National Medical Academy of Postgraduate Education

9, Dorogozhyzkaja str., Kyiv, Ukraine

Storozhchuk Yu. O., Assistant, Graduate Student of the Department of Radiology Shupyk National Medical Academy of Postgraduate Education 9, Dorogozhyzkaja str., Kyiv, Ukraine 